\title{
Genomic and functional analysis of gynecological carcinosarcomas
}

Anne-Sophie Sertier1, Isabelle Treilleux, Sandrine Boyault, Anthony Ferrari, Mojgan Devouassoux-Shisheboran, Janice Kielbassa, Laurie Tonon, Vincent Le Texier, Emilie Thomas, Anne-Pierre Morel, Roxane Pommier, Amandine Charreton, Anne Floquet, Florence Joly-Lobbedez, Dominique Berton-Rigaud, Gwenaël Ferron, Laurent Arnould, Sabrina Croce, Guillaume Bataillon, Pierre Saintigny, Eliane Mery-Lamarche, Christine Sagan, Ivo Gut, Fabien Calvo, Alain Viari, Isabelle Ray-Coquard, Alain Puisieux ${ }^{2}$

Introduction
Gynecological carcinosarcomas (GCS) are histologically
defined as biphasic neoplasms composed of an intimate
admixture of carcinomatous epithelial and sarcomatous
malignant components. Although these tumors represent a
small fraction of newly diagnosed uterine or ovarian cancers,
they disproportionately contribute to the population of
patients recurring and dying from disease. Due to their rarity
and to their histological complexity, genetic and functional
studies on GCS are scarce and the intimate mechanisms of
initiation and development of these highly aggressive and
heterogeneous neoplasms remain largely unknown.

\section{Carcinosarcoma dataset}
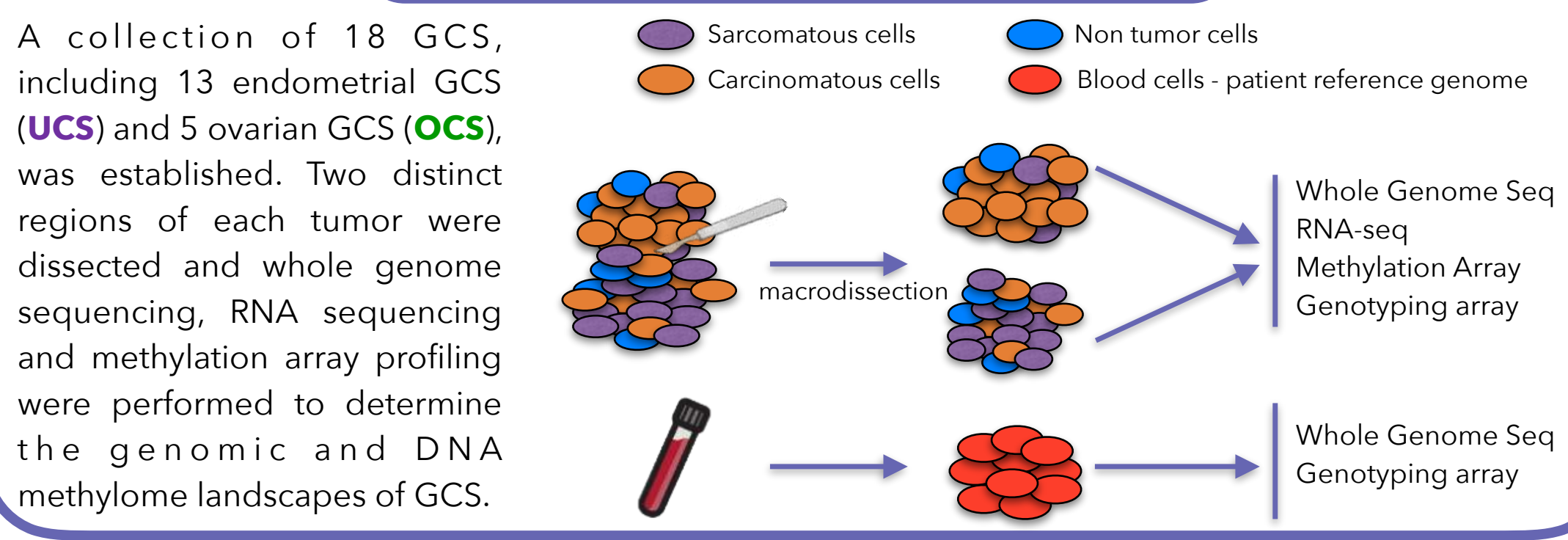

\section{Molecular characteristics}

\section{Structural variants}

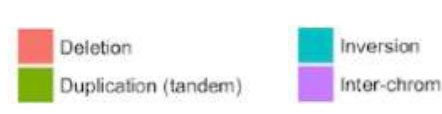

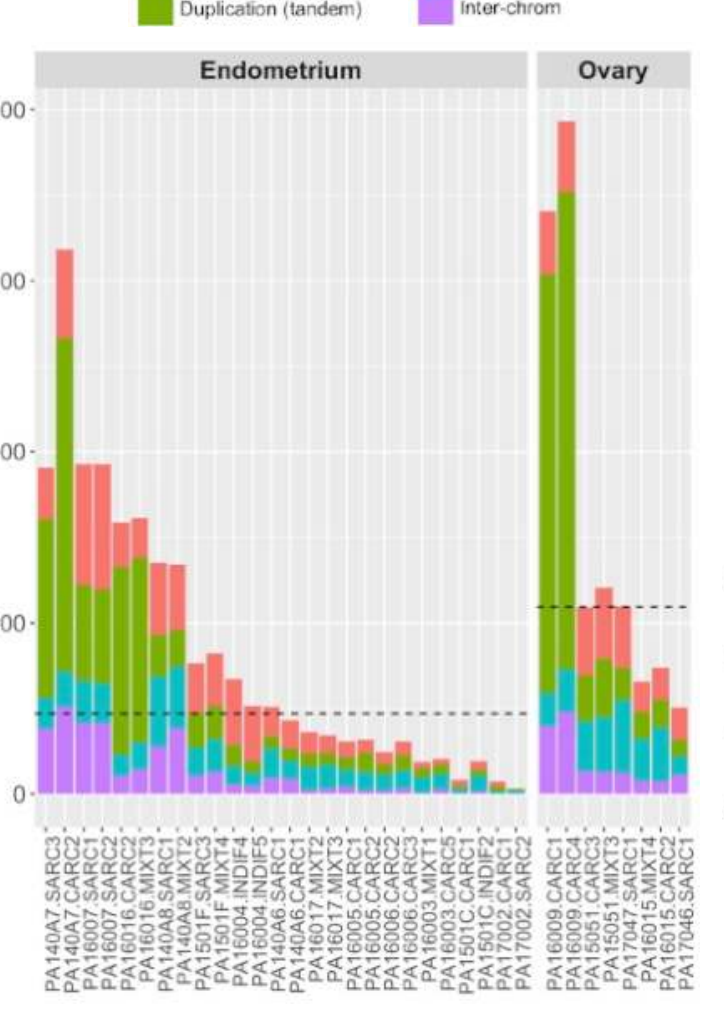

Single nucleotide variants

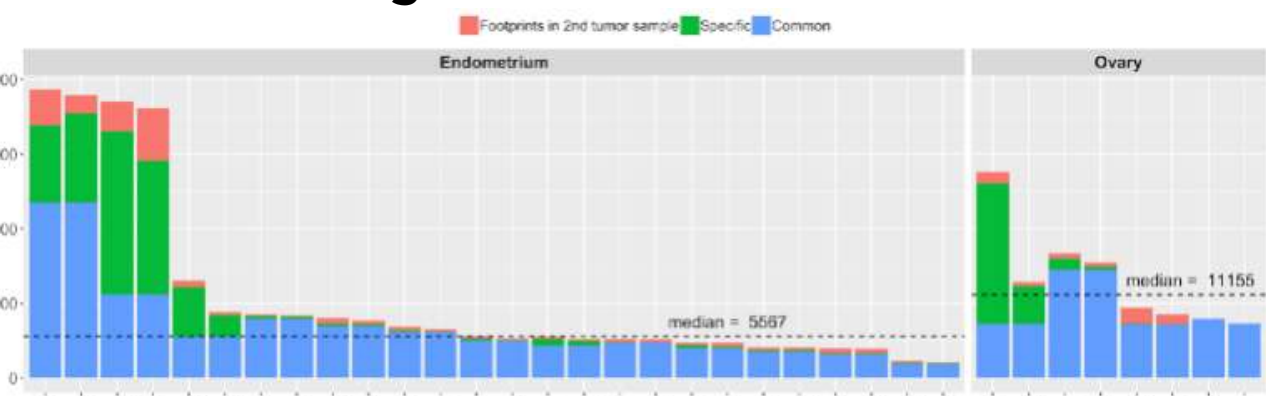

Small insertion/deletion variants

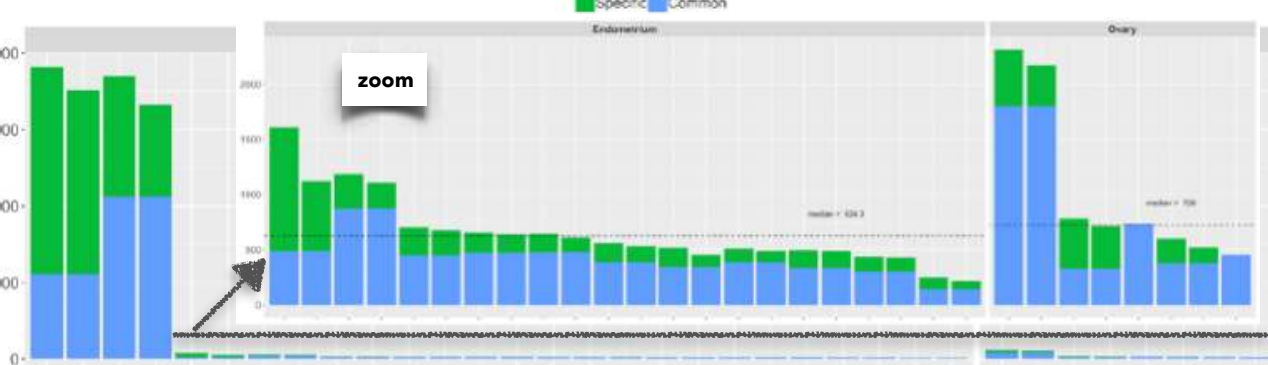

\section{Mutational signatures}

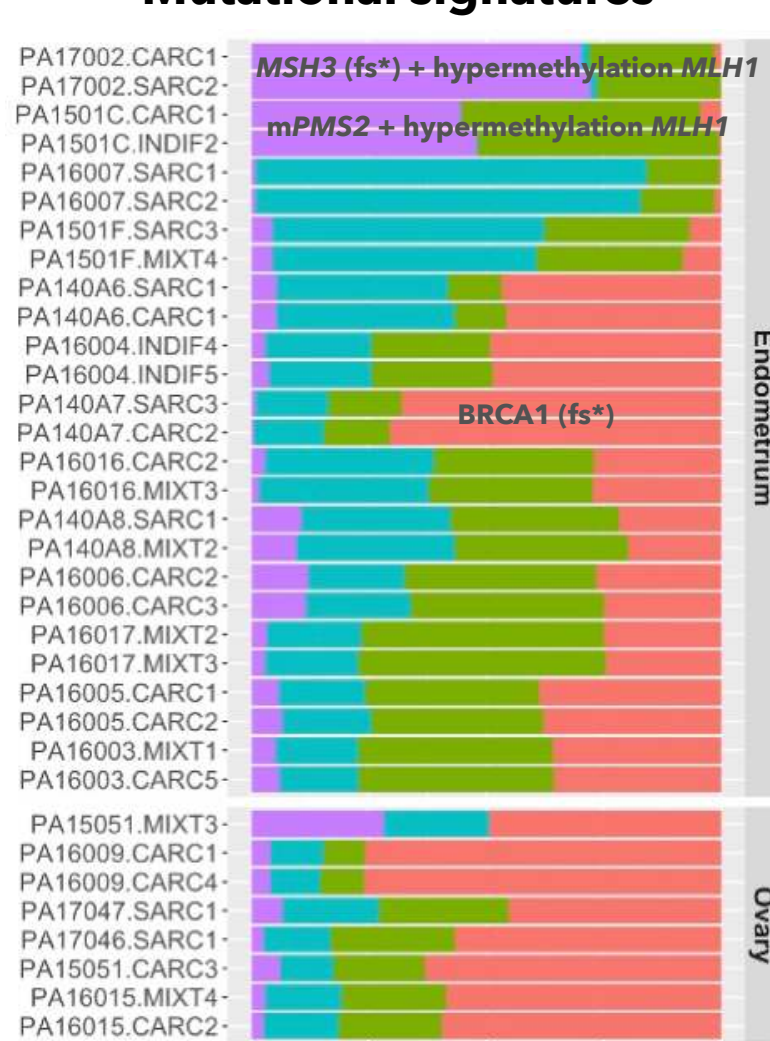

APOBEC: Cytidine BRCAness

Age : Endogenous $\quad$ MSI : Mismatch

\section{Results}

The evaluation of evolutionary

histories of GCS in 5 tumors

with pure carcinomatous and

sarcomatous elements

demonstrated that these

elements shared many somatic

alterations (mutations,

variants and copy

establishing unambiguously

the common genetic origin of these malignancies.

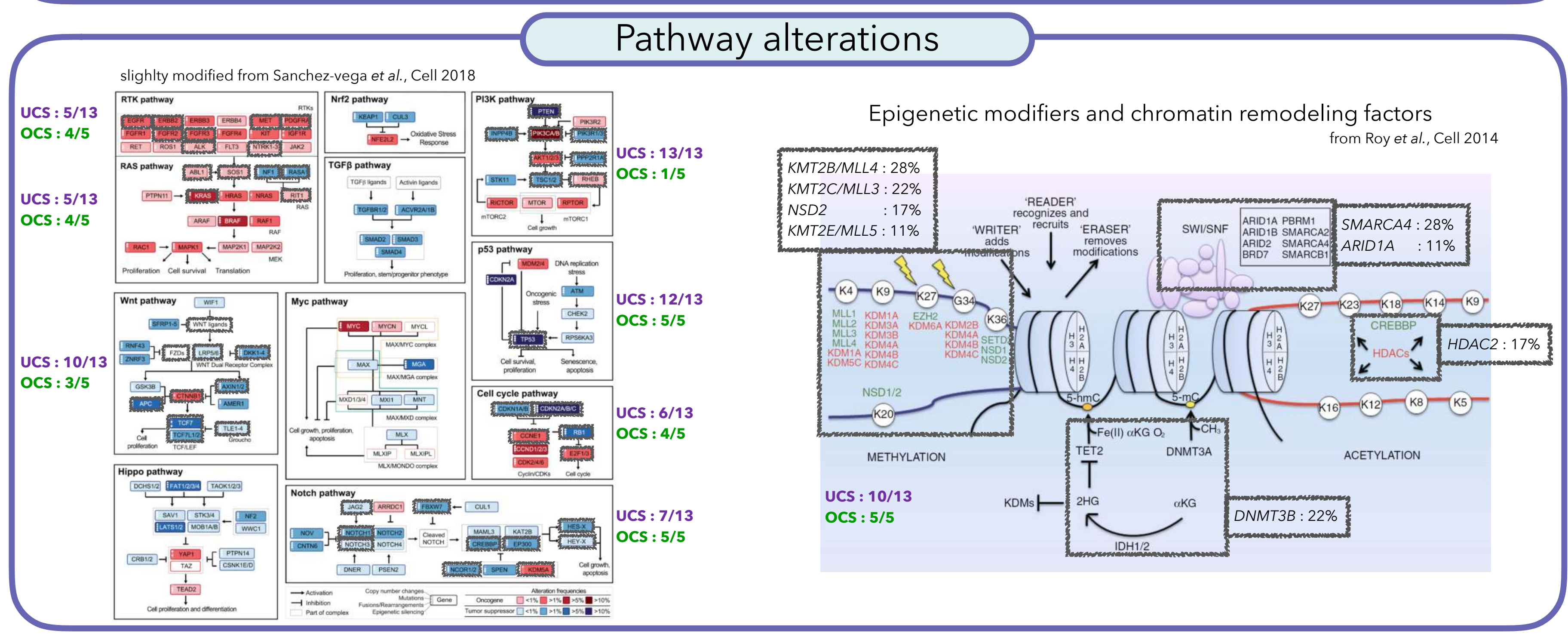

Differential methylome analysis

miR200c and miR141 are methylated in sarcoma components and are

demethylated in carcinoma components

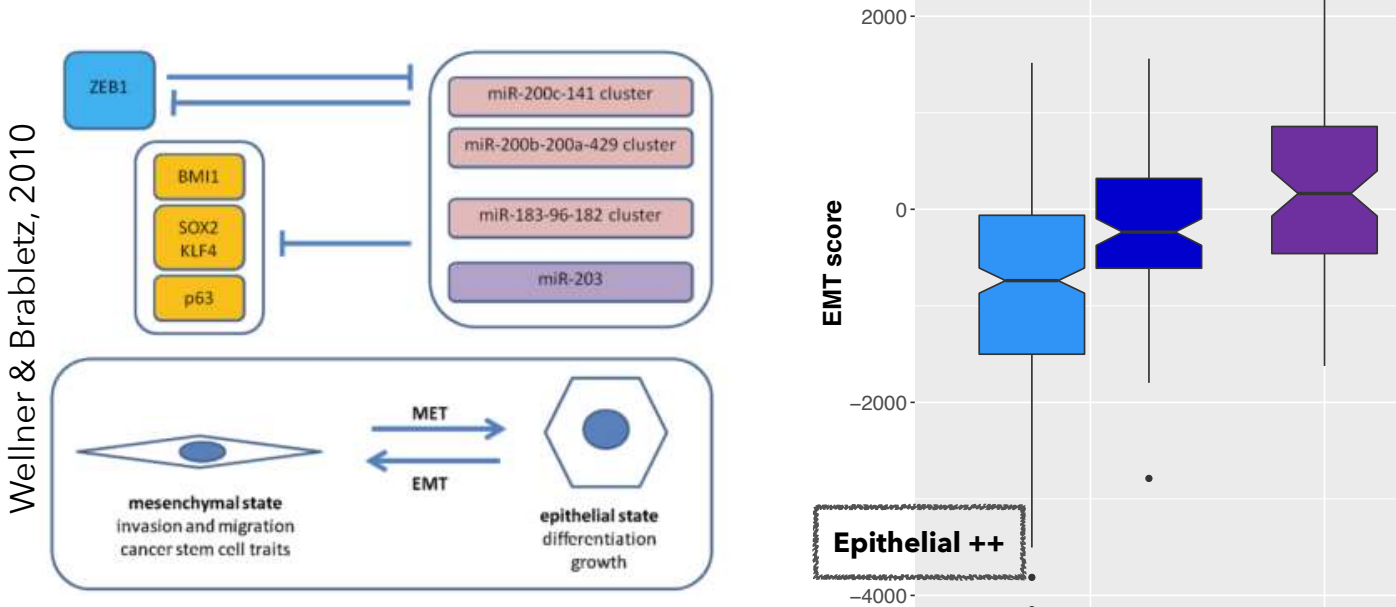

EMT process in Carcinosarcomas

$$
\text { Co-occuring alterations: }
$$

$$
\text { triade [TP53 + PI3K pathway + Epigenetic regulators] }
$$

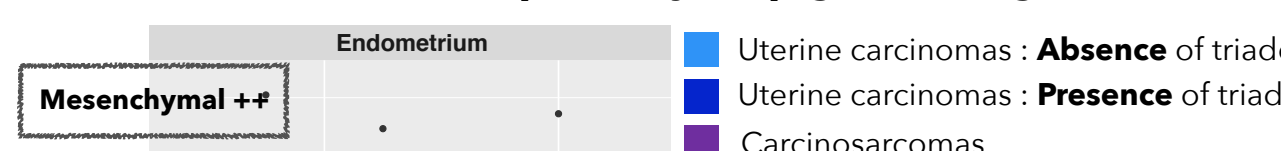

EMT score is computed with ssGSEA algorithm on SARRIO EMT gene lists (Sarrio et al. ,2008)

Uterine carcinomas with the triade [TP53+PI3K pathway+ epigenetic regulators] (26\%) show an EMT signature.

\section{Conclusion}

The most prominent mutations in GCS were TP53 mutations, mutations affecting the PI3K pathway genes and mutations affecting chromatin remodeling genes. The finding that root mutations in GCS are also frequent in gynecological carcinomas (compared from TCGA) strongly supports an epithelial origin of GCS with a progressive evolution relying upon a transdifferentiation process. This dynamic notion implies that carcinosarcomas are the end result of an evolutionary process and that early forms of GCS may be histologically classified as carcinomas.

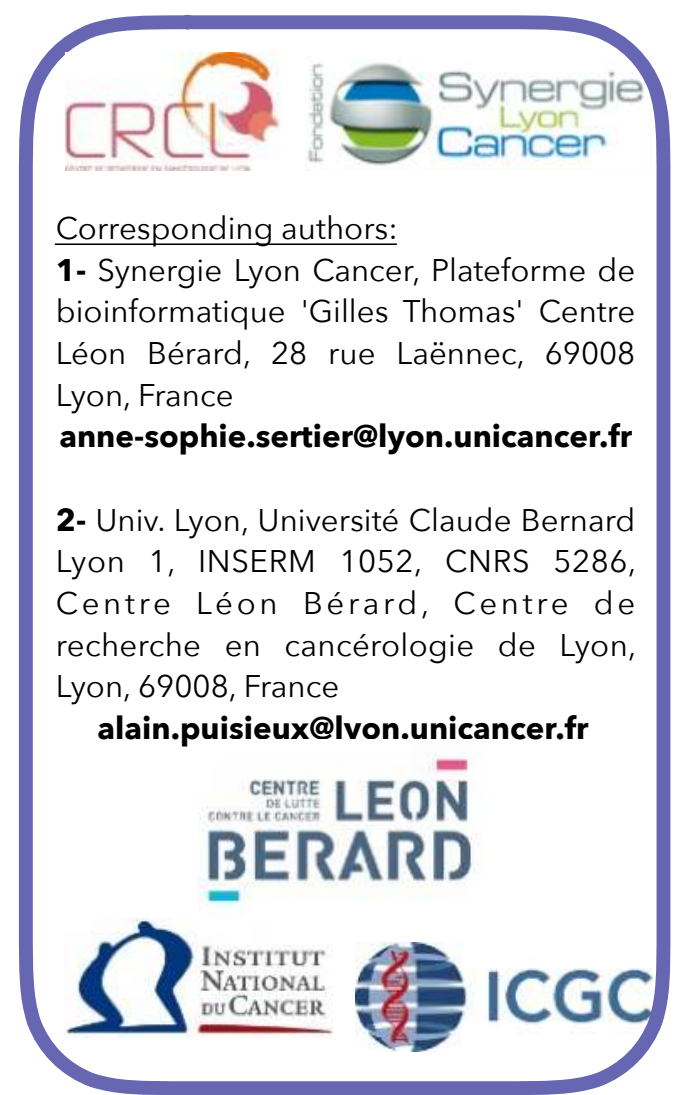

